\title{
The Role of Five-Letter Words in Literature: An X-Area in New Translation Studies
}

\author{
Alireza Akbari \\ Faculty of Foreign Languages, University of Isfahan, Iran \\ dictogloss@gmail.com \\ Masoud Shahnazari \\ Department of English Language and Literature, Kerman Branch, Islamic Azad University, Kerman, Iran \\ shahnazari@att.net

\section{Neda Aghaei Khorasgani}

Department of Foreign Languages, Khorasgan (Isfahan) Branch, Islamic Azad University, Isfahan, Iran Aghaee.neda@gmail.com

\section{Doi:10.5901/mjss.2015.v6n1s1p490}

\begin{abstract}
Taboo or in general term 'forbidden words' plays a key role in literature through times. Heretofore, Translation of taboo or forbidden words in literature is a pivotal task to do. According to Allan and Burridge (2006), "taboo refers to a proscription of behavior for a specifiable community of one or more persons, at a specifiable time, in specifiable contexts (p. 11)." Taboo words in New Translation Studies (NTS) amplifies its significant influence in source-target amalgamation. This is due to the fact that translations either source-based or target-based tend to be homogenized so as to transfer the near essence of the text. In this connection, the way of translating taboo in literary texts is of crucial importance. Most of the translators resort to the natural equivalence or one-to-one correspondence for the ease of the target reader. Translating in accordance with the source regulations or the target ones is not the absolute license dealing with taboo or forbidden words. Translators should consider the void or X-area between the source and the target language in order to decipher the underlying potential in Source-Target translation. This study seeks to clarify the meaning of this X-area in New Translation Studies in literary phrases and texts. To better understand it, this article scrutinizes four utmost important approaches. The intended approaches are (1) language persistency, (2) language sensitivity, (3) the role of the decoding ability, and (4) Equimediation paradigm which inspect equivalents either deeply or superficially so as to persuade the audience on their workability, feasibility, and practicality.
\end{abstract}

Keywords: Taboo, source-target amalgamation, natural equivalence, language persistency, language sensitivity, the role of the decoding ability, Equimediation paradigm.

\section{Introduction}

Translation of taboo-derogatory-words is the most challenging way in Translation Studies. Taboo words illustrate a generation gap amongst the elders and the youths. The way of speaking and the situation utilized are completely different in this direction. Every language along with particular traits explicitly exploits taboo words to show its power and degree of frequency of the intended words. Every taboo word has its own power of influence on the hearer. Some of them are utilized in jokes and colloquial speeches and they cause the hearer to enjoy hearing them. On the other hand, some others show insulting facets of the language and they make the hearer react to such derogatory words critically. Therefore, expressing such words besieges some constraints so that the speaker should be aware of them.

Let us first clarify the real meaning of taboo. Taboo derives from the intended Tongan word "tabu" whose originality refers back to the end of the eighteen century. The exact meaning of taboo is to show something which is thoroughly forbidden to exploit. Cook (1967) argues that

Taboo is the common expression when anything is not to be touched, unless the transgressor will risque some very severe punishment as appears from the great apprehension they have of approaching anything prohibited by it. In some cases it appears to resemble the Levitical law of purification, for we have seen several women who were not allowed the use of their hands in eating but were fed by other people. On enquiring the reason of it at one time they said that one of 
the women has washed the dead body of the chief already mentioned who died at Tonga, and another who had assisted was in the same predicament, though then a month after the circumstance had happened. It also serves as the temporary law or edict of their chiefs, for sometimes certainly articles of food are laid under restriction, and there are other circumstances regulated in the same manner as trading \& when it is thought necessary to stop it (p. 948).

As indicated above, taboo words are culture-bound and have nothing to do with other cultures. It is noteworthy to expound that utilization of taboo words requires determining the red lines between the speaker and the hearer. Importantly, politeness and respection are the opposite poles of taboo words. Therefore, the type of the generation either younger or older in this sense is of crucial significance. The speaker should recognize the type of the hearer so as to utilize such taboo words. So, the role of decoding ability (explained later throughout this paper) in taboo expressions is highly recommended. In this respect, the speaker has to scrutinize the sorts of situations. Frozen situations, consultative situations, and formal situations require being polite. On the other hand, relationships amongst the friends, pals, and chums are not much important to be maintained formally. In the latter case, the speaker somehow is allowed to use taboo words to break the ice.

In the shed of these expatiations, some questions are posed in this respect. How can the renderer/translator or the interpreter convey the exact sense of the intended taboo words in the target language? How can the translator be able to persuade the reader on workability of taboo words in source language? And how will it be possible for the translator to prepare the situation in order to reconcile the taboo words of source language to the target one? These questions will be answered throughout this paper in detail.

Translation of taboo words in films and serials are also more significant to ruminate. More specifically, taboo words in films differ greatly from the intended street talks in general. Media mass production should regard the type and the age of the audience in order to persuade either source or target watchers. In this respect, the translator should be aware of the fact that mistranslation of taboo words is vulnerable for the infants and their ilks. This suggests that the renderer should get familiar with the emotional aspects of the intended groups as well. In some cases, to create taboo persuasion on the reader or watcher, the translator/interpreter should tone down the concealed meaning of the taboo words in target language. This implies that degree of frequency and potency on the intended words should also be taken into account.

Last but not least, the rationale behind focusing on taboo translation stemmed from the condition that taboo words would be treated differently across cultures. This is because one cannot build the stable framework of the intended term particularly in the field of Translation Study concentrated on source and target language culture to see the similarities and differences. To gap this filling, this paper proposes four factors to simulate the simultaneous situation in the target language in order to tone down the obscenity of taboo words and saturate the taste of target audience. The intended factors include language persistency (inner circle or deep layer of language), language sensitivity (outer circle or surface layer of language), the role of the decoding ability (the type of encountered audience), and Equimediation paradigm. More specifically, this study inspects how these factors could be applied to translating taboo words in various situations and their impact on the target audiences. And eventually, this paper opens up the new window on how to quench the visibility impact and bearing of five letter words-taboo words- in target translations.

\section{Types of Taboos in Literature}

Allan and Burridge (2006, pp.5-8) argue that there are five kinds of taboos which cover some special criteria of language in order to show their particular functions in particular situations under investigation. The intended taboos will be scrutinized by the translational regulations so as to show their sterility or fertility in the target language. These categories are as follow:

\subsection{Fatal Taboos}

In nineteen century, there was a superstitious creed in that it depicted the demonic or diabolic potency of tabooed objects comparable with the robust and vulnerable power of the Polynesian chief or the emperor of Japan. The so-called power addressed the antediluvian and credulous people aiming at putting people's lives into jeopardy. For example, Mead (1937) argued fatal taboo as "to describe prohibition against participation in any situation of such inherent danger that the very act of participation will recoil upon the violator of the taboo (pp.502-5)" It is inferred that human being is curious in nature. His/her curiosity put him/her in danger. Human seeks to experience everything and he/she wants to reach to the sole destination per se. However, human beings insist on any factor which prohibits him/her from doing or experiencing something. Taboo words act like the flamboyant material which causes to shimmer on the human's eyes. For instance, in 
Islamic regions, whenever a married woman has an affair with a man followed by a sexual intercourse, she is sentenced to death. Or as another example, in old Hawaii; whenever a bourgeois has sex with his sister, he puts himself into fatal death. Therefore, one has to conclude that fatal taboos are completely culture-bound and they show the people's beliefs or creeds of special region of the world. Thus, dealing with these kinds of fatal taboos requires paying special attention in that they can rouse the anger of society. Irrespective of target language function, translator should transmit the real sense of the fatal taboos because of the fact that the renderer should be faithful to the function of source language context. Somehow, function of source language can be coincided to target language contexts. Translator can simulate the similar source position in target language. In this kind of situation, the renderer utilizes the interpretive resemblance [direct rendering] (Gutt, 1991, p.122) in translation. Furthermore, the translator can exploit the technique of covert version (House, 1997, p. 161) of source text to transfer the near essence of the intended term into target language.

\title{
2.2 Uncleanliness Taboos
}

These sorts of taboos cover physical contacts such as body squirting, menstruating women, and body masturbation. For instance, Orthodox New York Jews shuns sitting beside the menstruating women. They deem that menstruating woman is ominous and she pollutes the man. They think that mensuration woman has not the right of being in public and she should purify herself next to the men. As another example, corpse in many communities postulates something unclean in that nobody is allowed to touch the corpse. Should anyone touch the corpse of the human or the cadaver, she/he cannot permit to handle the food. In this vein, translator faces so many problems and is challenged to deal with these taboos. Translating the intended taboos is equal to playing with the people's creeds. Therefore, the renderer should translate them in accord with source language regulations in order to show their impacts on source contexts. In this connection, the translator or the interpreter as the mediator should incline more on source language so that she/he can transfer the near sense of the intended taboo words in target language context.

\subsection{Violating Taboo and Getting Away with it}

Hobley (1910) expounds that:

\begin{abstract}
It sometimes happens, however; that a young man unwittingly marries a cousin; for instance, if the part of the family moves a way to another locality a man might become acquainted with a girl and marries her before he discovered the relationship. In such a case the thahu [or ngahu, the result of the violation of the taboo] is removable, the elders take a sheep and place it on woman's shoulder, and it is then killed, the intestines are taken out and the elders are solemnly sever them with a sharp splinter of wood.... and announce that they are cutting the clan 'kutinyarurira', by which they mean that they are severing the bond of blood relationship that exists between the pair. A medicine man then comes and purifies the couple (p. 438).
\end{abstract}

As inferred, the translator should tone down the power of taboo words in some situations and then turns them mild or pleasant in target language context. It is the wrong belief that the renderer can remove the intended taboo words to avoid some possible problems during the course of translating. "Getting away with it" does not mean to eliminate the taboo in translation. The skillful translator utilizes the technique of toning down in translation. Cutting and diminishing the intended values or impacts in translation can transfer the underlying meaning of source language context. Translator in any conditions should convey the hidden or concealed meaning of source text. Persuasion of the target reader is completely coincided to the deep layer of source context.

The ultimate aim of translation in this category will be to ameliorate the rate of offense and insult so as to make the mild and pleasurable situation at which the reader understands the hidden layer of source language context.

\subsection{Less Serious Taboos}

These kinds of taboos will not deteriorate the situation between the speaker and the hearer. It does not have corporal punishment, dead penalties, incarceration, and social eviction. They are mostly spoken by the young generations to show their intimacy. These sorts of taboos belong to a particular ilk of people feeling a sense of intimacy and affection with one another. For instance, regulations of the law by the family or country might be regarded as down-graded taboos. That is due to the fact that the whole set of people might be treated as the family and it does not cause serious outcoming despite its disapproval. Norms and conventions might be behaved as less serious taboo unless they are treaded appropriately in the society. Therefore, less serious taboos are more common in this connection. Translator in this 
category is free to opt for various equivalents of the intended taboo. She/he can choose either from source or target language contexts to show their vastness of equivalents. But, it should be noted that the renderer as the mediator should not be inclined to one particular pole (source or target languages). She/he should walk on the straight road and line between the two poles simultaneously in order to persuade the reader on the workability of the chosen equivalents. The suitable way in this category is to simulate the lesser taboo in target language context which will be tangible for target reader. Satisfying the needs of the reader is the ultimate aim of Translation Studies. Generally speaking, the role of the translator in taboo translation might be to recreate, represent, and to reproduce the same situation in target language. In some cases, translator resorts to transcreate and she/he seeks to decipher one stable role to deal with and in other situations she/he just translates the intended part to convince the reader for the way it is. Transcreation and Translation are two indispensable parts in New Translation Studies.

\subsection{Imperfect Taboos}

Every taboo word has an underlying part which is completely unreachable from source or target user. Taboo words are not absolute enough to inspect. They sometimes suffer from being in void and suspense situations. That is due to the fact that the user of the intended taboo will not be able to carry them out in the direct and appropriate situations. For instance, Voltaire (1694-1778) had an affair with his widowed niece in that he wrote: "My child, I shall adore you until l'm in my grave.... I would like to be the only one to have had the happiness of fucking you, and I now wish I had slept with no one but you, and had never come but with you. I have a hard on as I write to you and I kiss a thousand times your beautiful breasts and beautiful arse." As implied, the translator cannot render one-to-one correspondence (natural equivalence). That is due to fact that natural equivalence paradigm will not be suitable in this essence. Every word of this speech has a concealed intention which the translator should consider as the pivotal or heart of the dialog. Therefore, translator has to transfer the real and hidden intention of source text thoroughly. In this kind of situation, the renderer might be better to resort to directional paradigm in translation to show the feasibility and workability of source equivalents in the target language. Every equivalent is relative and the translator is not sure of its complete practicality of the intended equivalent in target language. Nevertheless, it is suggested that translator is better to get familiarized with target language structures and orders. One-to-one correspondence and one-to-two correspondence are just parts of the scene. The real duty is to distinguish which kind of correspondence is suitable in target language. For instance, for less taboo words the translator is allowed to utilize the natural or one-to-one correspondence in that she/he can simulate the intended situation into target language. But for the imperfect taboos, translator should exploit directional equivalence or one-to-two correspondence in that she/he pursues to transfer the real intention of source language context.

\section{Discussion}

Every translator has his/her own approaches when dealing with taboo words in source text. Some translators make use of keeping the real and original version of the intended taboo into target language context. Yet, others prefer to modify the underlying version of the taboo words and then turn them to a less degree of potency due to the fact that they consider the type of the target language audience. This paper aims at exploring some particular approaches in source taboo translation so as to make target translation more tangible and pleasurable in this regard. To achieve this, firstly; translator should exploit them (e.g. language persistency, language sensitivity, and the role of the decoding ability) into his/her translation and secondly, situate him/herself in x-areas (e.g. Equimediation paradigm) in order to decipher some shared parts between source and target languages. In this respect, proximity to source and target languages is of crucial importance to convey the exact and real essence of the taboo words/expressions. These approaches are as follow:

\subsection{Language Persistency}

Language persistency alludes to particular situation in which people eschew using source item in target language context. That is due to the fact that patriotism plays a crucial factor in most languages. Every language has its own background of language items. Most of the followers prefer to utilize nativized words in order to be understandable enough for all. However, some of the non-nativized words enter target languages which are thoroughly inevitable in nature. Henceforth, the duty of the translators is to prepare and obtain particular situation deeply so as to make the intended words nativized and workable. Amongst these languages, the German and Italian languages shun using non-nativized words because of their language heritage. In this case, the translator should reconcile source context to target one and discover some common grounds between the two poles in translation. Translator as the mediator should conglomerate source language 
to target one. Despite its arduousness, it is completely possible to achieve. Simulation of the real intention of the intended taboo words in the target language is of great importance in translation. For instance, "SMS" in the English countries are the most prevalent words. But, the intended word in the Persian language does not gain its popularity in this connection. Most of the Persian people prefer to use the Iranian equivalent. Therefore, the Persian morphologists recommend utilizing the equivalent as "Payamak or Peyqame Kutah". The intended word becomes popularized in accord with the system and regulation of the Persian and Turkish languages as well. As another example, in the novel of Lady Chatterley's Lover, Giuolio Monteleone translated one part of the source translator's note in the Italian language in order to show language persistency in the target language.

\begin{abstract}
"Le battute effetivamente in dialatto, sono state tradotte in italiano. Non si poteva altrimenti, salvo ricorrere a uno dei nostril dialetti. Ma ne sarebbe nato alcunché di risibile (Monteleone trans., 1960, p.211)"

"Gloss: These lines are actually in dialect but have been translated into standard Italian. They could not have been translated otherwise, except by resorting to one of our own dialects. Had that been the case, the result would have been laughable."
\end{abstract}

The translator notices to use some special items in the target language in order to show that the Italian people mostly prefer the nativized words. In the above translator's note, the translator utilizes the term as "effetivamente" in the target language. While the source language exploited the adjective term as "actual" instead of "actually". The translator understands the taste of the Italian reader that he utilizes the technique of modulation so that he can persuade the reader on its workability. Or as the other example, the translator perfectly shows the temporal distance in the target language translation such as "ricorrere" which means "to resort". That is due to the fact that the intended term implies anaphoric referencing which is suggestive of temporal distance. Thus, utilizing the terms either taboo or formal expression requires considering the taste of the target reader in general. Language persistency should be treated with cautious. This is because of the fact that language item is the rudimental part and it constitutes the foundation of the society. Deepness of equivalents in this category is highly recommended. For instance, Muslims prefer not to eat/consume pork in that they believe that pork contains some obnoxious elements which are pernicious for their bodies. Therefore, they prefer to eat Halal meat such as beef, fish, and so forth. Or Jews believe that foods especially meat should be conformed to the regulations of Kashrut. Foods which may be consumed in accord with the Kashrut refer to "Halakha" in Hebrew language. It is better to say that translator can render dietary taboos into some medical treatment. For example, Muslims shun consuming insulin as it is emanated from pork derivation. So, the translator instead of using pork derivation, she/he prefers to recommend insulin as the lawful medications. In this vein, the renderer should lessen the degree of taboo's effect deeply in target language. Violation of taboo means violation of target language norms and conventions and it causes translation to be treated as artificial as possible. The reason behind this notion is that language persistency and taboo's impression are mutually exclusive in target language.

\title{
3.2 Language Sensitivity
}

The function of language sensitivity is much alike to the language persistency. However, there exists one vital difference between them. The language persistency works and scrutinizes the language items in the deep layer of language related to the particular beliefs, taboos, and creeds and they are institutionalized deeply in the language. Whereas, the language sensitivity alludes to superficiality of language and consequently it is prone to change. The translator prior to the act of translating should carry out some research on practicality of language persistency and then decorates his/her translation as language sensitivity in this connection. To translate taboo words in target language, translator should consider some aspects of taboo words in target context. The first factor is "euphemism" defined as to lessen the degree of derogatoriness of source item in target language. It is the art of translator to diminish some facets of source items and then turns them into target values which are quite acceptable in target language. For instance, instead of expressing "é morto da infarto"; translator diminishes the degree of intended insult and renders it as "morì per attacco cardiaco". In this case, target reader as a judge of translation can connect easily to the intended rendering. This is due to the fact that most of target readers seek to peruse whatever they like. And it is the task of the translator to satisfy the needs of target reader as much as possible. The second factor in taboo translation is "dysphemism". Dysphemism can be defined as an offensive connotation about the denotatum. Denotatum alludes to the time when the language expressions such as denotation are used. For instance, "bow-wow" or "gee-gee" all denote a canine animal of either sex. But, the connotations are much more different to that of their denotations. The intended words imply the baby-talk for the word of "horse". And finally, the last factor is orthophemism. Orthophemism can be expressed as neutral expressions or straight talks which 
are not dulcet-sounding in this connection. For instance, the English example as "he eats avocado" can be rendered as "mangia la pera alligatore" in the Italian language to show its orthophemistic-based meaning of the source item. As an exhaustive instance, "faeces" (orthophemism) can be rendered as "poo" (euphemism), and "shit" (dysphemism) in target language in accord with various genres. The degree of potency and offense range from lesser to higher. Therefore, translator should consider the power spectrum of the intended words throughout translating. Needless to say, the ultimate goal of the translator as the mediator is to reconcile source language to target one so that she/he can meet the needs of reader. Saturating the needs does not mean annihilating some readers' creeds and beliefs. The translator at any span of time has to defer to the readers' expectations. For example, inter-racial marriages are regarded as the taboo in some communities. Fortunately, with the advent of cultural homogenization, the intended item is treated easily amongst societies. In this respect, translator as an expert is able to connect source people to target one via ST-TT amalgamation. Sharing the common grounds between people is highly respectable in New Translation Studies. People in source language and target prefer to be in between. As another example, "Negro" in the English language refers to someone having the black skin. The intended word is regarded as one who is downgraded in the society. The Persian rendering of this item mostly utilized in comic situation is as "Kaka Siah". This equivalent postulates something funny in the mind of the Persian speakers or readers. But, in America this refers to a taboo word and the translator should translate this item superficially as "Blackie" in the English language especially Australian language not to insult target reader. In the Italian language the term "fare i complimenti" is the prevalent structure/item which the translator should treat it cautiously. In most of the western countries, people don't compliment with each other upon the requests, invitations, or food serving. While, in some eastern countries, people eschew accepting the first time invitation and they wait for the second time compliment in this regard. In this kind of situation, the translator first should be familiar with the language patterning (background) of target and source languages (Akbari, 2014b) and then she/he should know how to lessen the degree of compliment in target language. Therefore, "fare i compliment" in western countries might be rendered as "the one time invitation or request" which considers more palpable for the target reader.

\subsection{The Role of the Decoding Ability}

Nida (1964) argues that the role of decoding ability might be defined as:

(1) The capacity of children whose vocabulary and cultural experience are limited. (2) The double standard of capacity of new literates, who can decode oral message with facility but whose ability to decode written message is limited. (3) The capacity of average literate adult, who can handle both oral and written messages with relative ease; and (4) the unusually high capacity of specialist (doctors, theologians, philosophers, scientists, etc.), when they are decoding messages within their own areas of specialization (pp. 156-71).

Prior to the act of translating, the translator has to distinguish the types and the sorts of audience. In this sense, translator should resort to the purpose paradigm which aims at satisfying the needs of target reader. The role of the client in this category is of high importance. The main axis of purpose paradigm is the commission or reader's instruction. The translator will be treated as an expert who seeks to empower the target side of language context thoroughly. Decoding ability is just one factor of translation in the circle of purpose paradigm. Other factors are target-sidedness, target reader intention, and the translator as the expert. All of them should be considered as parts of the team. Daniel Gouadec (2007) argues that source and target language contexts, translator, and the client are like a team who should interact with one another in order to create the suitable translation for target reader. He uses the terms as "job specification" in which the client gives some instructions [commission] to the translator. Should the translators find out the completeness of the specifications, they can render source language. However, if the intended specifications are not complete enough, the translators should be able to seek further details. Therefore, before the act of translating, the translator should prepare the pre-translation so that the reader can negotiate on the appropriateness of the intended equivalents, structures, and the way of thinking of translation in target language text. And finally, agreement amongst the players in the text is the core element in translation. For instance, "ghoul" is the neutral term in the English source language. Should the translator seek to satisfy or nativize the intended taboo for the children, he/she has to utilize the term as "bogyman" to make it tangible so as to transfer the exact sense of the taboo into target language. For the ilk of children in taboo translation, the translator must resort him/herself to orthophemism in order to defer the taste of the children. Straight-talks and neutral speaking are the two factors which should be observed in this category. As another example, "Christ" is the taboo term used for particular group as vernacularists. This is the due to the fact that Christ covers the dysphemism degree of transference and then translator has to render the term "Jesus" as "Christ" for special group of people. In the Italian language, two 
interactants should never say "Ciao" to each other. "Ciao" should be said in special situation among the friends, or peer groups. The intended term in formal conversation is treated as "Buongiorno" in that it conveys the overt prestige of the conversation. In this vein, translator should first consider the context of situation and second she/he must regard the degree of participation (audience) in target language. "Ciao" should be rendered as "cheerio" in the English language to transfer the vernacular [informal] situation amongst interactants. In all these cases, the task of the translator is to decipher the common parts between source and target languages and then tries to homogenize the intended rendering as well. Walking on the void areas in translation requires understanding source-target language patterning. This makes translation more palpable for the reader seeking to know the regulations of source language into target one. Accordingly, considering the type of people in New Translation Studies is highly recommended. The other most problematic example in all nations is the word usage of "sex". Most of the families do not directly speak about "sex" in public situation. This is due to the fact that the intended term in all cultures devotes its limitations for special group of people. "Sex" has the big and meaningful connotational aspect of meaning. No one is allowed to speak about it in every situation. However, some nations break the laws of the intended term and express their opinions directly in front of others. It means that they lessen the degree of harshness of the word "sex" in their societies. In this connection, connecting source language to target one requires utilizing some strategies such as euphemism, loss and gain, and so forth. Most of the Iranian translators prefer to render such term as "rabete" to lessen the degree of its derogatory and accordingly decoding ability considers this term as the neutral one in target language as well.

In the light of these expatiations, prior to the act of translating; translator is better to know the taste of the target reader. The reason behind this notion is that the ultimate aim of translation is to conglomerate all types of people together. Conglomeration means that the translation should be in between. Translation is treaded as something as third part or third space to link the first part (source) to the second one (target).

\subsection{Equimediation Paradigm}

Equimediation is the blending term composed of equivalence and intermediation. Unlike equivalence and purpose/descriptive paradigms treating equivalence source-sidedness and target-sidedness respectively, Equimediation paradigm treats the equivalence in-between source and target languages. Prior to the act of translating, translator as the mediator should situate him/herself in the gray spot explained more in continuation of this study. The final aim of the translator is to conglomerate the deep-surface layers and source-target reconciliation with each other to satisfy the needs of either source or target readers. In every field of study, there are some gray spots neglected by the researcher. These spots contain some pivotal and uncharted information which are able to direct the outcome of the research. In Translation Studies, these spots would be forgotten and yet they have not been used to clarify and divulge the extraordinary relations and factors.

In order to clarify the main essence of Equimediation paradigm, some new terms are explained in the following sections.

\subsubsection{Weak equivalents}

It refers to any equivalents which are less frequently used and more likely to be forgotten. These kinds of equivalents are exposed to be cliché and temporarily utilized in either source or target languages such as cliché metaphors, allegories, slangs, expressions, allusions, simile, and temporal conversational slangs. For instance, avoid like the plague, acid test, alive and kicking, and Achilles heel.

\subsubsection{Strong equivalents}

These sorts of equivalents are not exposed to change, that is due to the fact that the backgrounds of the intended equivalents are richly valuable in nature. Cultural terms such as adages, proverbs, moral maxims, fixed expressions, frozen expressions, and collocations are categorized in this group. For examples, no pain no gain, "Always do what is right", the mule has more horse sense than a horse, and you can push people just so far.

\subsubsection{Brittle equivalents}

These types of equivalents are the most sensitive equivalents in target language. Sensitivity exists in cases which are completely pertained to the belief, creeds, and personality of people. This category should be taken cautiously in New 
Translation Studies. The important fact is that brittle equivalents sometimes incline to source and sometimes to target language contexts. This is possible when target and source readers are completely aware of their language patternings and try to find some ways to connect them together. Euphemism (sweet talking), dysphemism (speaking offensively), and orthophemism (straight talking), and derogatories are in this category. For instance, loo, poo, Christ, Jesus, Lord, cur, and dopy.

Equimediation paradigm uses such deep-superficial equivalents explaining the root and core of either source or target language contexts. New Translation Studies seek to amalgamate the two poles in translation. Shaded areas in translation consist of some rudimental and fundamental facets of translation which need to be taken as granted. Translation is like crossing the road. It means that translator should be between two lines of water so as to pass the river. In translation, the renderer should consider the nature of source text in order to clarify the nature of target language. Translator acts like the catalyzer seeking to connect source to target language contexts. Connecting the two bridges should be carried out cautiously because the risk of falsifying source text is at high stake. Unlike passing the river, translator should hybridize source to target languages to find out the common points in these poles. Therefore, inclination to source and target languages should be performed equally at the same time. Whether translator inclines more to either source-target or deep-surface, she/he will walk on the rim of falsification issue in translation.

To clarify the function of Equimediation paradigm in translation, it is worth revealing one exhaustive figure so that the translator convinces to ascertain the fundamental facets of this paradigm.

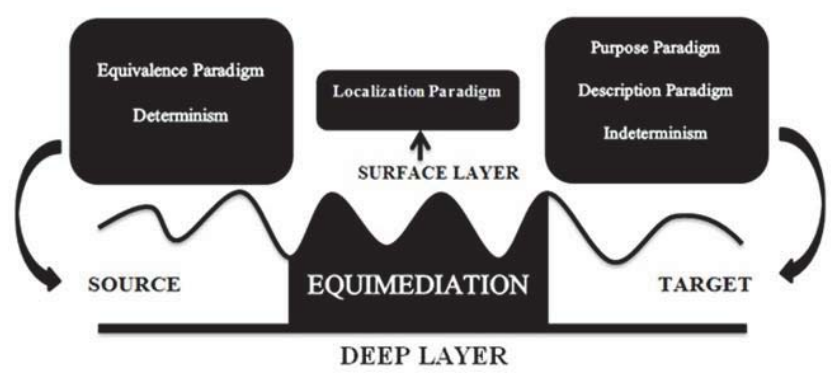

Figure 1: Equimediation Paradigm

As indicated, Equimediation is the kind of paradigm inspecting equivalents into two rudimentary factors: (1) SourceTarget Amalgamation and (2) Deep-Surface Layer. These items act as key roles in New Translation Studies which aims at clarifying the deep and surface layer of the translation either in the source or target language contexts. It is notable that strong equivalents incline more to the deep layer of source-target amalgamation and the weak one more on surface layer of source-target amalgamation. Brittle equivalents sometimes incline to be strong enough and sometimes to be weak equivalents in this direction. Brittle equivalents are contextual-temporal-based in which they are used in different contexts along with various purposes.

Let's give an example to reveal the real function of Equimediation paradigm. Goddard (1995) encounters translating one title of novel in the target language. He utilizes one unbelievable technique to transfer the main essence of the source language text. His technique is in accord with the Equimediation paradigm. Most of the translators are not aware of the fact that translation involves some various equivalents which are completely applicable and feasible for the target reader. It is better to note that translation of titles differs from the text per se. Every title covers some aspects of equivalence paradigm including more than one equivalents in the target language. Goddard translates the French novel as the source language into English rendering. The name of the novel is "L'amer". During the translation of the intended novel, translator pays attention to the fact that "L'amer" can be rendered by more than one equivalents in the target language. Goddard seeks to establish an agreement amongst the source, target languages, client, and the translator. Therefore, he infers that the title includes other equivalents such as "mere (mother)", "mer (sea)", and the "amer (bitter)". In order to show the feasibility of the translation of the title into the target language, he utilizes the schematic figure to transfer the translation impossibility. This schematic figure is as follows: 
As inferred, this scheme contains some source-target equivalents namely: (1) These Our Mothers, (2) The Sour Mothers, and (3) The Sea Smothers. The term Mother and Mere share some common parts in translation. They emanate from the same source. They are categorized in partial wafting in New Translation Studies. Akbari (2014a) defines wafting as "a direct and intense translation process, it has a connection with rendering or transferring as the original SL by inserting or absorbing the SL words or phrases form into the RL's in accordance with prestigious, frequency, and intensity of the intended words (p. 27)." In this example, 'mere' and 'mother' are treated partially and they carry the same sense with each other in source-target translation. This technique puts translation in between. Translator can exploit source-target amalgamation terms in his/her translation.

In these equivalents, the translator uses some various but related equivalents so as to convey the main essence of the text. This translation shows the degree of relationships amongst the surface, deep, source, and the target language contexts. In this situation, the reader is the decision maker who can judge about correctness and understanding of the target text. Importantly, in the circle of translation, translator and the client are the two decision makers respectively. New Translation Studies let the reader decide about feasibility and practicality of the target text.

For instance, the three expressions "Go to hell", "Fuck off", and "Get your ass out of here" are all share the same deep function and meaning in the target language. This is due to the fact that the usability of these terms are solely relied upon the situation and environment operated under deep-surface amalgamation. Heretofore, the translator in these occasions can simulate the similar situation in the target language.

As another example, the term as "Shithouse" might be rendered as "das Scheißhaus" in the German language. However, the intended rendering does not make sense for the English learner who wants to learn German as the second language. In this connection, the translator resorts to the Equimediation paradigm and then determines the borderline between the two equivalents and then conglomerates them. In this essence, the renderer uses the terms as "Scheißhouse" or "Shithaus" to persuade the reader on their workability. In this regard, the reader is free to judge about the meaning and underlying inference of the intended equivalents in the target language. As another example, "a clumsy person" might be translated as "Der Klutz" in the German language so as to show the border between the source and the target languages. In the English language, the intended term might be utilized by the term as "clod" as partial wafting. However, the translator modifies the equivalent phonologically in order to show the amalgamated line between the two equivalents (Akbari, 2013). In all instances, translator seeks to decipher the common grounds between source and target contexts.

It can be expressed that in most of the cases; translator is able to exploit the technique of Source-Target amalgamation in order to reconcile the two intended poles in translation. It is better to say that Equimediation paradigm differs from the blending or shortening techniques in linguistics. The latter refers to the process of shortening the term in L1 or L2. On the other hand, the former utilizes both L1 and L2 altogether without considering the superficial aspects of source and target language.

Translator should always consider the taste of reader so that he/she can persuade reader on the feasibility of the equivalents. Nevertheless, it is the skill of translator to resort to weak, strong, or brittle equivalence in different situations. In translation of taboo, translators mostly resort to weak and brittle versions of Equimediation paradigm. This is due to the fact that taboo/forbidden words are mostly spoken by some particular groups in a society. Therefore, translator should be faithful to this group as well.

\section{Conclusion}

Taboo words always exist in all societies in order to show the sense of insult or friendliness. In some cases, they address the people's creeds and sometimes express the character of people. Dealing with these kinds of norms in highly developed, developed, or less developed communities is completely different with one another. People's inferences are mostly different in these societies. Therefore, taboo or forbidden words are culture-bound terminologies. In this regard, the task of translator is of great importance. The translator is always between the two poles in her/his translation: source and target language contexts. She/he should act cautiously so as to transfer the exact and near meaning of the intended taboo words. The ultimate aim of translator is to persuade the reader on feasibility, practicality, and workability of the equivalents chosen in target language. Equivalents in target language might not be treated as the natural equivalents. 
That is due to the fact that one-to-one correspondence is not the sole factor when translating such taboo words. The translator is better to know the terse etymology of the forbidden words as he seeks to convey its essence into target contexts.

In the long run, translators were trying to establish the stable framework dealing with culture-based words such as forbidden/taboo words. However, the proposed suggestions were not successful enough to corroborate the nature of taboo words in source language context. Generally speaking, most of the approaches expressed by translators are also culture-bound and they are not able to position in between. Translator as the mediator should decipher some factors and approaches being in-between source and target language contexts. This is because of the fact that the area between source and target languages shares most of commonalities between in translation. Thus, this study proposes four approaches in taboo/forbidden words translation. The first approach is language persistency scrutinizing the intended equivalents deeply so as to transfer the underlying parts of equivalents in target language. The second approach is language sensitivity used for superficial intention of the equivalents. Superficial means the decoration of target language through stylistic traits. It is noteworthy to say that the chosen equivalents should be dulcet enough for target reader so that she/he wants to continue perusing the text. The third approach is the role of the decoding ability. The task of translator is to satisfy every need of target reader (TR). TR consists of all ilks of people in a society. Therefore, she/he should render in such an understandable way so as to saturate all needs of the TR. And the last approach is Equimediation paradigm which a translator can distinguish the commonalities between source and target language contexts. It is somehow pertained to Source-Target Amalgamation. The purpose of Equimediation paradigm is to connect source pole to target one in order to fill the void gaps between them. The gap between source language and target one can solve most of the translation problems. Therefore, New Translation Studies should consider this empty-void-area so as to corroborate the nature of Source-Target Amalgamation.

\section{References}

Allan, K. and Burridge, K. (2006) Forbidden Words: Taboo and the Censoring of Language. Cambridge: Cambridge University Press.

Akbari, A. R. (2013) Exploring into the New Model Procedure in Translation: Wafting as a Case in Point. International Journal of Education and Literary Studies, (1) (1), 19-20.

Akbari, A. R. (2014a) Challenges in Translation of Proper Nouns: A Case Study in Persian Translation of George Orwell's Animal Farm. International Journal of Comparative Literature and Translation Studies, (2), 19-28.

Akbari, A. R. (2014b) The Perks of Politeness Translation Strategies. Theory and Practice in Language Studies, (4) (6), 1193-1200.

Cook, J. (1967) From the Original MMS in J.C. Beaglehole, (eds.) The Journals of Captain James Cook, (3): The Voyage of the resolution and Discovery 1776-1780. Cambridge: Cambridge University Press.

Goddard, B. (1995) A Translator's Diary, in Simon, S. (eds.) Culture in Transit: Translation and the Changing Identities of Quebec Literature. Montreal: Vehicule Press.

Gouadec, D. (2007) Translation as Profession. Amsterdam, Philadelphia: Benjamins.

Gutt, E.A. (1991) Translation and Relevance: Cognition and Context. Oxford: Blackwell.

Hobley, C. W. (1910) British East Africa: Kikuyu Customs and Belief. Thahu and its Connection with Circumcision Rites. Journal of the Royal Anthropological Institute of Great Britain and Irelandvol (40), 428-52.

House, J. (1997) A Model for Translation Quality Assessment. Tübingen: Gunter Narr.

Mead, M. (1937) Encyclopedia of Social Sciences. London: Macmillan.

Nida, E. A. (1964) Towards a Science of Translating. Amsterdam: Leiden: E. J. Brill.

\section{Notes}

Authors' Created Term "Taboo"

Corresponding Author 